\title{
AdS black holes with higher derivative corrections in presence of string cloud
}

\author{
Tanay K. Dey ${ }^{1, \mathrm{a}}$, Subir Mukhopadhyay ${ }^{2, \mathrm{~b}}{ }_{\mathbb{D}}$ \\ ${ }^{1}$ Department of Physics, Sikkim Manipal Institute of Technology, Sikkim Manipal University, Majitar, Rongpo, East Sikkim, Sikkim 737136, \\ India \\ ${ }^{2}$ Department of Physics, Sikkim University, 6th Mile, Gangtok 737102, India
}

Received: 16 July 2020 / Accepted: 18 October 2020 / Published online: 2 November 2020

(C) The Author(s) 2020

\begin{abstract}
We consider asymptotically AdS black hole solutions in Einstein Gauss Bonnet gravity in presence of string clouds. As in the case of black hole solutions in Gauss Bonnet gravity, it admits three black hole solutions in presence of string clouds as well within a region of the parameter space. Using holography, we have studied the quark-antiquark distance and binding energy in the dual gauge theory.
\end{abstract}

\section{Introduction}

Study of strongly coupled gauge theories remain a challenge due to lack of appropriate systematic formulations and machineries. On that score, the AdS/CFT duality has been proved to be quite a useful route. This duality implies that $S U\left(N_{c}\right), \mathcal{N}=4$ Super Yang-Mills (SYM) theory living on the boundary of the one higher dimensional space time in the limit of strong coupling is dual to a weakly coupled gravity theory in the higher dimensional space time and vice versa [1-4]. It has been found that there is a correspondence between the black hole configurations in the gravity theory and the dual boundary theory in its deconfined phase. On the other hand, the confined phase of the dual gauge theory is dual to pure AdS space time configuration. The transition [5] between the pure AdS configuration and the black hole configuration has been interpreted as equivalent to the transition between the confined and deconfined phases of the dual gauge theory living on the boundary.

A more realistic scenario is to consider a gauge theory with fundamental quarks and baryons, which are absent in $S U\left(N_{c}\right), \mathcal{N}=4 \mathrm{SYM}$ theory. In order to incorporate fundamental quarks, [6-10] consider cloud of hanging strings

\footnotetext{
a e-mails: tanay.dey@gmail.com; tanay.d@smit.smu.edu.in

b e-mails: subirkm@gmail.com; smukhopadhyay@cus.ac.in (corresponding author)
}

whose end points are tracing out the contour of the loop at the asymptotic boundary. In the dual gauge theory living at the boundary, the end points of the string correspond to the quark and anti-quark, whereas the string itself corresponds to the gluons.

Several works [11-17] have considered string clouds which amounts to considering external heavy quarks in the dual gauge theory. The Einstein theory with string clouds admits black hole solutions with three different radii and the thermodynamics of them has been discussed by one of us [12], which also showed a Hawking-Page like phase transition between two black hole solutions. Such three black hole solutions along with the phase transitions have been showed in $[18,19]$ in the context of Einstein Gauss Bonnet (GB) theory in absence of the string clouds. Thermodynamics of black hole solutions in Einstein Gauss Bonnet theory in presence of strings clouds has been extensively discussed in [13]. Effect of the strings clouds on the thermodynamics of the extended phase space of Gauss Bonnet black hole has also been discussed in [15].

In this work we have considered the black hole solution of Gauss Bonnet gravity in presence of string clouds and discussed thermodynamics as well as its effect on the dual gauge theory. In a similar model [20,21], found that there is an upper bound for the quark-antiquark $(Q \bar{Q})$ separation for large black hole and thus identified it with the deconfined phase. Along the similar line, we have studied the $\mathrm{Q} \bar{Q}$ distance, as well as the binding energy for the black hole solutions in GB theory with string clouds. We find the screening length of $\mathrm{Q} \bar{Q}$ pair increases as the temperature decreases and though for the small black hole, it can go to a larger value compared to that of large black hole, there remains an upper bound. Therefore one can conclude that the present model does not admit a confined phase. The value of the binding energy is more negative in the case of the large black hole compared to that of the small black hole showing $Q \bar{Q}$ bound 
state in the case of large black hole is more stable. In particular, at high enough temperature, the $\mathrm{Q} \bar{Q}$ distance vanishes showing that the $\mathrm{Q} \bar{Q}$ bound state does not exist.

The paper is structured as follows. In Sect. 2, a brief discussion of the black hole solution in presence of external string cloud is included. We then discuss the thermodynamical quantities and their behaviour in Sects. 3 and 4 respectively. Thereafter in Sect. 5 we check the instability of $Q \bar{Q}$ bound state by studying the depth of the U-shape string hanging from boundary of the asymptotically AdS background. Finally we study $Q \bar{Q}$ bound state and its binding energy at low temperature in Sect. 6. At the end we summarize our work in Sect. 7.

\section{Black hole solution}

We begin with a brief review of black hole solutions [13-17] of Einstein Gauss Bonnet theory, which are asymptotically AdS in presence of string clouds. The string clouds correspond to introducing strings in the background. They are extended from the boundary to the horizon of the black hole or the center of the space time. The higher derivative terms can be thought of as leading to subleading correction in the 't Hooft coupling.

The $(4+1)$ dimensional gravitational action along with Gauss Bonnet terms is given by

$\mathcal{S}=\frac{1}{16 \pi G_{5}} \int d x^{4+1} \sqrt{-g}\left(R-2 \Lambda+\alpha L_{G B}\right)+S_{m}$,

where $G_{5}$ represents the gravitational constant. $R$ is the Ricci scalar and $\Lambda$ is the cosmological constant. We write $g_{\mu \nu}$ as the space time metric tensor and $g$ represents its determinant. The term

$L_{G B}=R^{2}+R_{\mu \nu \rho \sigma} R^{\mu \nu \rho \sigma}-4 R_{\mu \nu} R^{\mu \nu}$,

is the higher order correction term due to the quantum fields renormalization. This term is called Gauss-Bonnet correction in the Lagrangian. The $\alpha$ is the coefficient of the GaussBonnet counterpart which plays a crucial role in this theory.

In order to incorporate the string clouds we have added a further term, $S_{m}$. Since $S_{m}$ represents a large number of the strings its contribution can be written as,

$S_{m}=-\frac{1}{2} \sum_{i} \mathcal{T}_{i} \int d^{2} \xi \sqrt{-h} h^{\beta \gamma} \partial_{\beta} X^{\mu} \partial_{\gamma} X^{\nu} g_{\mu \nu}$.

where we have Integrated over the world-sheet, $h^{\beta \gamma}$ is the world-sheet metric and $\beta, \gamma$ correspond to the world sheet coordinates. $S_{m}$ is the contribution of a large number of strings. We denote the tension of $i$ 'th string by $\mathcal{T}_{i}$.
We will assume that the strings are uniformly distributed over the three spatial directions and write the density as

$a(x)=T \sum_{i} \delta_{i}^{3}\left(x-X_{i}\right), \quad$ with $a>0$.

With this assumption, as shown in [13-17] the action (1) admits a black hole solution. The metric tensor of this solution is,

$d s^{2}=-g_{t t}(r) d t^{2}+g_{r r}(r) d r^{2}+r^{2} g_{i j} d x^{i} d x^{j}$.

Here $g_{i j}$ is the metric on the $(4-1)$ dimensional boundary and

$g_{t t}(r)=1+\frac{r^{2}}{4 \alpha}\left(1-\sqrt{1+\frac{32 \alpha M}{r^{4}}-\frac{8 \alpha}{l^{2}}+\frac{16 a \alpha}{3 r^{3}}}\right)=\frac{1}{g_{r r}}$,

where $l$ corresponds to the AdS radius and related to cosmological constant via the relation, $\Lambda=-\frac{6}{l^{2}} . M$ is the constant of integration, which represents the mass of the solution.

The solution is singular at $r=0$, which is cloaked by a horizon located at $r_{+}$, which can be obtained by setting $g_{t t}\left(r_{+}\right)=0$,

$1+\frac{r_{+}^{2}}{4 \alpha}\left(1-\sqrt{1+\frac{32 \alpha M}{r_{+}^{4}}-\frac{8 \alpha}{l^{2}}+\frac{16 a \alpha}{3 r_{+}^{3}}}\right)=0$.

To get the real solution of the above equation, the discriminant should remain positive which implies following condition,

$r_{b h}^{4}\left(1-\frac{8 \alpha}{l^{2}}\right)+16 \alpha\left(2 M+\frac{a r_{b h}}{3}\right)=0$,

to be imposed on the maximum value of the horizon radius. We can trade the ADM mass of the black hole solution for the radius of the horizon using the following relation,

$M=\frac{3 r_{+}^{4}+3 l^{2} r_{+}^{2}-2 a l^{2} r_{+}+6 l^{2} \alpha}{12 l^{2}}$.

This solution is asymptotically AdS and it is necessary to analyse the stability of this solution towards a decay into the pure AdS. We will consider a thermodynamic analysis of it in the next section. As we will see, for a given temperature it admits three different kinds of black holes along the line of [18] and we will study the relative stability of these solutions.

\section{Thermodynamics}

Black holes can be considered as thermodynamic systems and various thermodynamical quantities associated to black hole maintain a similar type of thermodynamical law as thermodynamic systems follow. In the present section we will consider the solutions and their different thermodynamic 
aspects. In what follows, we set the term related to gravitational constant, $16 \pi G_{5}=1$ and also consider unit volume of the 3-sphere.

- Temperature: To begin with the black hole temperature is given by,

$$
T=\left.\frac{1}{4 \pi} \frac{d g_{t t}}{d r}\right|_{r=r_{+}}=\frac{6 r_{+}^{3}+3 l^{2} r_{+}-a l^{2}}{6 \pi l^{2}\left(r_{+}^{2}+4 \alpha\right)},
$$

This gives rise to a cubic equation in $r_{+}$and for a given temperature, generically, we can expect three solutions for $r_{+}$.

- Entropy: Assuming that the first law of thermodynamics is satisfied by the black hole, we obtain the following expression for the entropy,

$$
S=\int T^{-1} d M=\pi\left(\frac{r_{+}^{3}}{3}+4 \alpha r_{+}\right) .
$$

- Specific heat: Specific heat can easily be obtained by using the formula $C=T \frac{d S}{d T}$ and is given by,

$$
C=\frac{\partial M}{\partial T}=\frac{\pi\left(r_{+}^{2}+4 \alpha\right)^{2}\left[6 r_{+}^{3}+3 l^{2} r_{+}-a l^{2}\right]}{6 r_{+}^{2}\left(r_{+}^{2}+12 \alpha\right)+2 a l^{2} r_{+}-3 l^{2}\left(r_{+}^{2}-4 \alpha\right)} .
$$

- Helmholtz free energy: Considering the energy of the black hole $E$ to be equal to its mass we get the following form of the Helmholtz free energy, $F=E-T S$ for these black holes.

$$
\begin{aligned}
F & =E-T S \\
& =-\frac{3 r_{+}^{6}-3 l^{2} r_{+}^{4}+108 \alpha r_{+}^{4}+4 a l^{2} r_{+}^{3}+18 \alpha l^{2} r_{+}^{2}-72 \alpha^{2} l^{2}}{36 l^{2}\left(r_{+}^{2}+4 \alpha\right)} .
\end{aligned}
$$

- Landau function: In order to study the transitions between different phases, we have constructed the Landau function computed around the critical point, where radius of the horizon plays the role of the order parameter. The construction goes as follows; first we consider a function $G$ as a power series in the horizon radius $r_{+}$,

$$
G\left(T, r_{+}\right)=\alpha_{0} r_{+}^{0}+\alpha_{1} r_{+}^{1}+\alpha_{2} r_{+}^{2}+\alpha_{3} r_{+}^{3}+\alpha_{4} r_{+}^{4}+\cdots,
$$

where $\alpha_{i}$ are functions of temperature $T$ and are chosen so as to satisfy the following conditions:
- Up to a certain temperature $T_{\min }, G$ will have only one minimum at $r_{+}=r_{+1}$ with $r_{+1} \geq 0$.

- Once the temperature is above $T_{\min }$ another minimum appears at $r_{+3}$. Minima at $r_{+}=r_{+1}$ and $r_{+}=r_{+3}$ must be separated by a maximum at $r_{+}=r_{+2}$. We demand that at high temperature, the second minimum is globally stable. In order to achieve this, we can consider only up to the quartic power of the order parameter and neglect all the terms, which are higher order in the order parameter. Therefore, the Landau function can be written as;

$$
G\left(T, r_{+}\right)=\alpha_{0}+\alpha_{1} r_{+}^{1}+\alpha_{2} r_{+}^{2}+\alpha_{3} r_{+}^{3}+\alpha_{4} r_{+}^{4} .
$$

- In order to determine these five $\alpha_{i}$ we impose the following five conditions.

- At the three extreme points the function should satisfy $\left.\frac{\partial G}{\partial r_{+}}\right|_{r_{1}, r_{2}, r_{3}}=0$,

- Expression of the temperature can be obtained from the condition of extrema of this function.

- Finally we get back the expression of free energy of the system by substituting temperature in the Landau function.

From the above five conditions we can compute the constants $\left(\alpha_{0}, \alpha_{1}, \alpha_{2}, \alpha_{3}, \alpha_{4}\right)$. The Landau function, when written in terms of temperature and the order parameter, reduces to the following expression,

$G=\frac{3 r_{+}^{4}-4 \pi l^{2} r_{+}^{3} T+3 l^{2} r_{+}^{2}-48 \pi \alpha l^{2} r_{+} T-2 a l^{2} r_{+}+6 \alpha l^{2}}{12 l^{2}}$.

An analysis of these thermodynamic quantities of the black hole and their dependence on various parameters of the model reveals a complex thermodynamic phase structure. This will enable us to study the issue of thermodynamic stability of the various solution admitted by the present model.

\section{Phases of black hole}

In order to explore the phase structures of the solutions, we will examine the behaviour of the various thermodynamic quantities with respect to variation of different parameters. Similar discussion of thermodynamics of Einstein Gauss Bonnet black hole in presence of string clouds has also appeared in $[13,15]$. For the sake of simplicity of notation, we scale a, $\alpha$ and the horizon radius with appropriate powers of 1 to render them dimensionless as follows:

$\bar{a}=\frac{a}{l}, \quad \bar{\alpha}=\frac{\alpha}{l^{2}} \quad$ and $\quad \bar{r}=\frac{r_{+}}{l}$. 


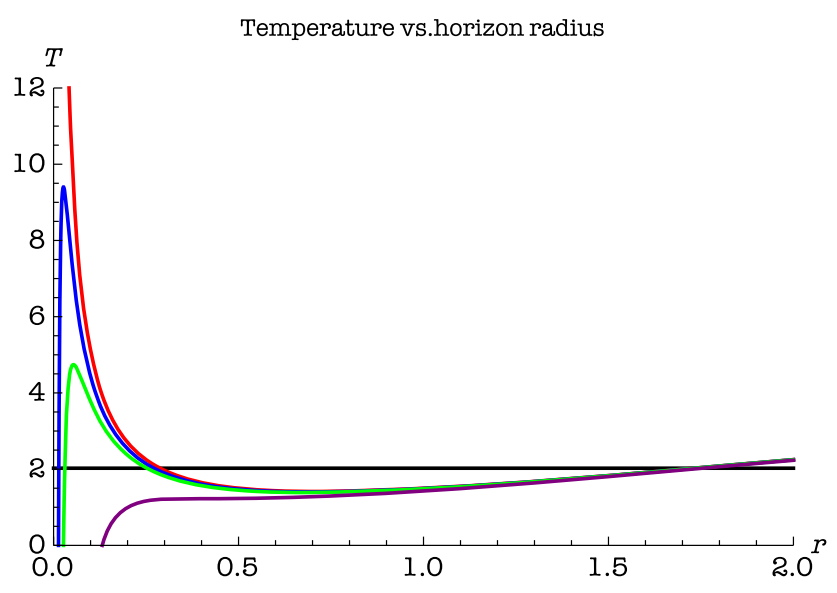

(a)

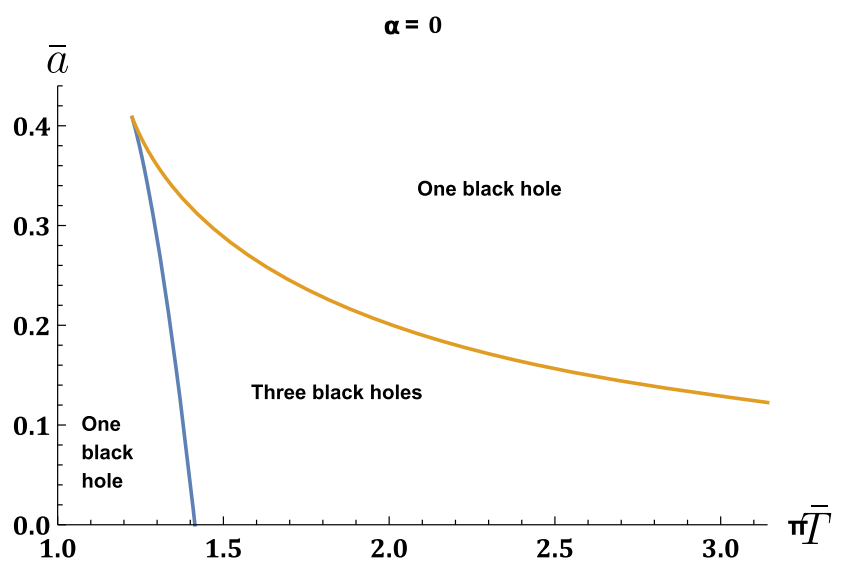

(b)

Fig. 1 Both the graphs are for $\bar{\alpha}=0$. a Temperature $\bar{T}$ vs. scaled horizon radius $\bar{r}$ and $\mathbf{b}$ string cloud density $\bar{a}$ vs. temperature $\pi \bar{T}$

We have introduced the temperature for the black hole solutions in (9), which can be expressed as

$\bar{T}=\frac{6 \bar{r}^{3}+3 \bar{r}-\bar{a}}{6 \pi l\left(\bar{r}^{2}+4 \bar{\alpha}\right)}$.

It shows that for a fixed temperature, the scaled horizon radius $\bar{r}$ satisfies a cubic equation. If the cubic equation admits three real and positive solutions there are three black holes or it will be only one black hole solution.

The discriminant of the cubic equation is given by,

$$
\begin{aligned}
\triangle=- & {\left[16 \bar{\alpha}(\pi \bar{T})^{4}+\frac{2 \bar{a}}{3}(\pi \bar{T})^{3}+\left(432 \bar{\alpha}^{2}-36 \bar{\alpha}-\frac{1}{4}\right)\right.} \\
& \left.(\pi \bar{T})^{2}-9 \bar{a}\left(\frac{1}{6}-4 \bar{\alpha}\right)(\pi \bar{T})+\frac{3}{4} \bar{a}^{2}+\frac{1}{2}\right] .
\end{aligned}
$$

In the above equation and in the following plots we have set $l=1$. In the region of the parameter space where $\Delta>0$, it admits three real solutions and otherwise there is only one real solution. First we have plotted the region for $\Delta>0$ for $\bar{\alpha}=0$ in Fig. 1 .

In the left subfigure of Fig. 1, we consider a set of values of $\bar{a}$, the string cloud density and plotted temperature against $\bar{r}$, the radius of the horizon scaled appropriately. We observe that for $\bar{a}=0$ (red curve), there are two black hole solution for any temperature greater than a minimum temperature. However once $\bar{a}$ gets some positive value but below a critical value $\bar{a}=\bar{a}_{c}=\frac{1}{\sqrt{6}}=.408$ (for details see [12]) there are certain range of temperature where three black hole solutions exist. Outside this range only one black hole solution exist. Once $\bar{a}$ is above its critical value (purple curve), for all values of temperature we get one black hole solution.

In right subfigure of Fig. 1 we plot string cloud density $\bar{a}$ against $\pi \bar{T}$. For a given value of string cloud density $0<\bar{a}<$ $\bar{a}_{c}$ there are two values of temperatures $\bar{T}_{1}$ and $\bar{T}_{2}$, such that the three black hole solutions exist in the region between
$\bar{T}_{1}$ and $\bar{T}_{2}$. For $\bar{a}=0, \pi \bar{T}_{1}=\sqrt{2}$ and $\bar{T}_{2}$ is extended up to infinity and for $\bar{T}>\sqrt{2} / \pi$, we get only two black hole solutions, which cease to exist as we decrease the temperature below $\sqrt{2} / \pi$.

As we turn on the string clouds, its density $\bar{a}$ becomes non-zero and $\bar{T}_{2}$ becomes finite. As the density of the string clouds $\bar{a}$ increases more and more, the upper bound for three solutions $\bar{T}_{2}$ keeps on decreasing. On the other hand, the lower bound $\bar{T}_{1}$ also decreases but at a much slower rate. Finally, when $\bar{a}$ reaches a critical value, $\bar{a}=\bar{a}_{c}, \bar{T}_{1}$ and $\bar{T}_{2}$ meet, indicating the merger of all the three black hole solutions. For $\bar{a}>\bar{a}_{c}$, there exists only a single black hole solution.

Once we turn on the higher derivative correction $\bar{\alpha}$, the region of the three dimensional parameter space corresponding to $\bar{T}, \bar{a}$ and $\bar{\alpha}$, which admits three black hole solutions are shown in the Fig. 2. As $\bar{\alpha}$ increases, the upper bound of the temperature for the existence of three black hole solution $\bar{T}_{2}$ becomes finite. For example, for $\bar{\alpha}=.001$ and $\bar{a}=0, \bar{T}_{2}$ becomes approximately $3 / \pi . \bar{T}_{2}$ keeps on decreasing with increase of $\bar{\alpha}$. The critical value of $\bar{a}$ denoted by $\bar{a}_{c}$, where the two bounds $\bar{T}_{1}$ and $\bar{T}_{2}$ meet, keeps on decreasing as $\bar{\alpha}$ increases and vanishes at around $\bar{\alpha}_{c}=.01386$. For $\bar{\alpha}>\bar{\alpha}_{c}$ there will be only a single black hole solution.

In the Fig. 3 we have plotted temperature with respect to $\bar{r}$ for two specific values of string cloud density $\bar{a}$ and GaussBonnet coupling $\bar{\alpha}$. It shows that at a particular temperature, the horizon radii of these three black hole solutions are of different sizes and we call them small, medium and large depending on their horizon radii.

For $\bar{T}<\bar{T}_{1}$ only a single black hole exists, whose radius decreases as the temperature decreases. This single black hole persists even at zero temperature, where the radius reaches its minimum value, which can be expanded in a power series of $\bar{a}$, 


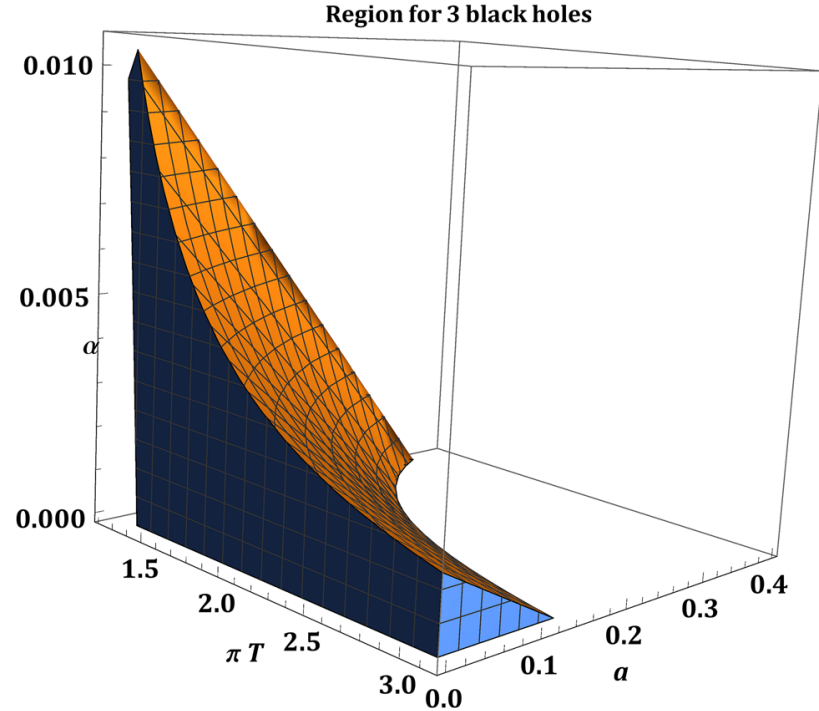

Fig. 2 Temperature vs string cloud density with higher derivative correction

$\bar{r}_{0}=\frac{\bar{a}}{3}+\mathcal{O}\left(\bar{a}^{2}\right)$

Having established the different black hole solutions from the study of their temperatures, we will enquire about their thermodynamic stability in the respective domains of the parameter space. For this purpose, we will examine the three different thermodynamic quantities: the specific heat, the Helmholtz free energy and the Landau function, that we have introduced in the earlier section.

We begin with the specific heat. The expression for the specific heat of the black hole solutions in terms of the three dimensionless parameters turns out to be;

$\bar{C}=\frac{\pi l^{3}\left(4 \bar{\alpha}+\bar{r}^{2}\right)^{2}\left[6 \bar{r}^{3}+3 \bar{r}-\bar{a}\right]}{6 \bar{r}^{2}\left(\bar{r}^{2}+12 \bar{\alpha}\right)-3\left(\bar{r}^{2}-4 \bar{\alpha}\right)+2 \bar{a} \bar{r}}$.

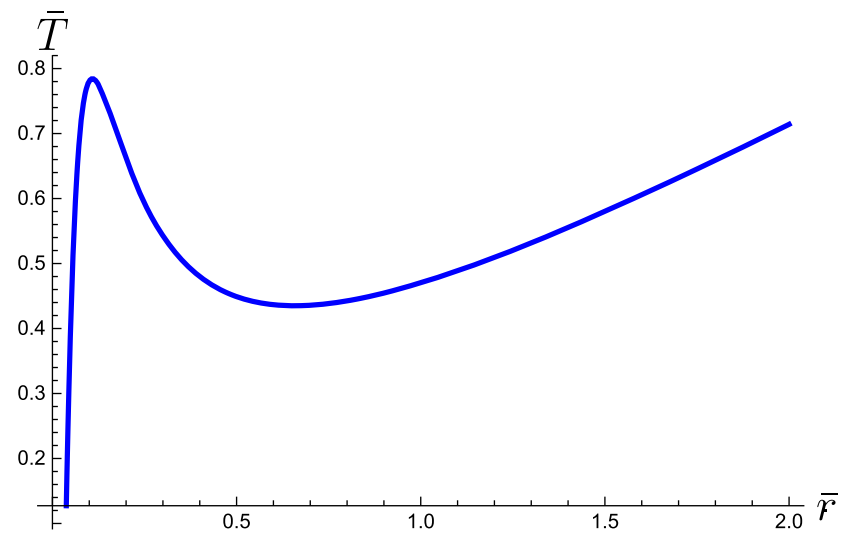

(a)
We have plotted the specific heat vs. temperature in Fig. 4b along with a plot of the horizon radii vs. temperature in Fig. $4 \mathrm{a}$ and we have chosen $\bar{\alpha}=0, \bar{a}=0.1$. In these figures, the red dashed line represents the small black hole which exists up to $\bar{T}<\bar{T}_{2}$ and as one can observe from Fig. $4 \mathrm{~b}$, its specific heat remains positive (though small) throughout the range where it exists. The blue line represents the medium black hole, which exists between $\bar{T}_{1}$ and $\bar{T}_{2}$ and the Fig. $4 \mathrm{~b}$ shows that the specific heat is negative leading to thermodynamic instability of the solution. The green, dotted line represents the large black hole. It starts off its existence from $\bar{T}=\bar{T}_{1}$ and persists for the entire range of temperature greater than $\bar{T}_{1}$. Once temperature is above $\bar{T}_{2}$ it represents the single black hole. As one can observe in Fig. 4b, it has a positive specific heat, showing it is thermodynamically stable. The behaviour of specific heat at the temperature, where unstable medium black hole meets the black holes with large horizon radius, indicates that the medium black hole decays at $\bar{T}_{1}$ through a first order phase transition.

Similar diagrams has been plotted in Fig. 5a, b for $\bar{\alpha}=$ $.004, \bar{a}=0.2$. As discussed earlier, due to turning on of the higher derivative correction, $\bar{T}_{2}$ has been reduced and approached $\bar{T}_{1}$ resulting the range of the temperature for the existence of the medium black hole smaller. The specific heat shows qualitatively similar features.

Next, we analyse the Helmholtz free energy, which can be written as;

$$
\bar{F}=-\frac{l^{2}\left[3 \bar{r}^{6}-3 \bar{r}^{4}+108 \bar{\alpha} \bar{r}^{4}+4 \bar{a} \bar{r}^{3}+18 \bar{\alpha} \bar{r}^{2}-72 \bar{\alpha}^{2}\right]}{36\left(\bar{r}^{2}+4 \bar{\alpha}\right)} .
$$

In the Fig. 6a, we have plotted the free energy vs. the temperature for $\bar{\alpha}=0, \bar{a}=0.1$. Since for our choice of parameters, the free energy of the small black hole is always less than that of AdS configuration, for small temperature

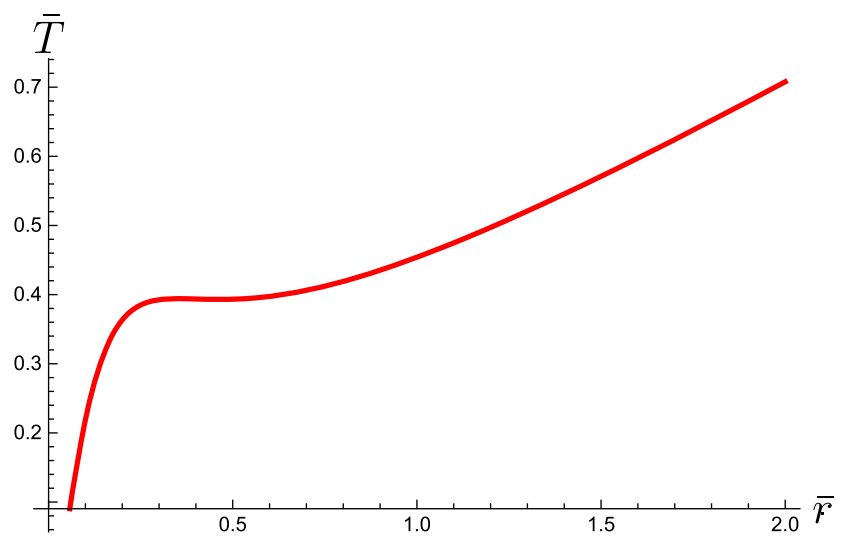

(b)

Fig. $3 \bar{T}$ vs. $\bar{r}$ plots. $\mathbf{a} \bar{a}=0.1, \bar{\alpha}=0.001$ and $\mathbf{b} \bar{a}=0.1, \bar{\alpha}=0.01$ 


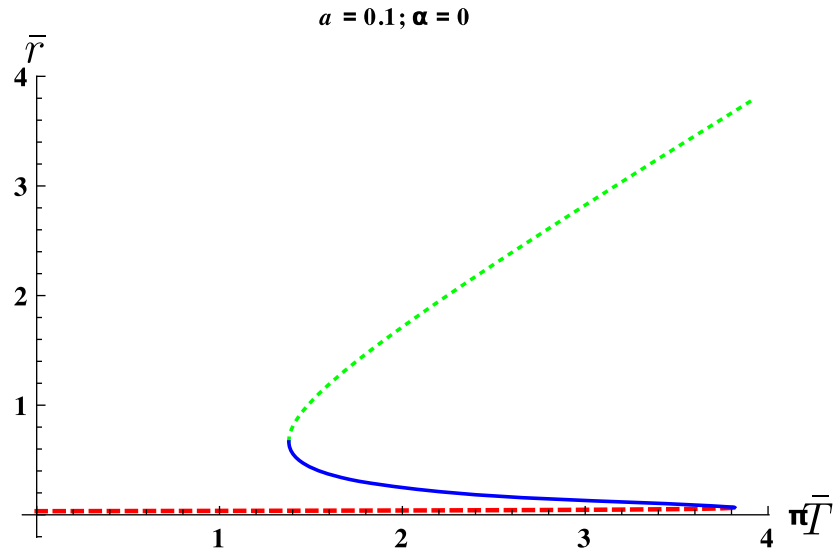

(a)

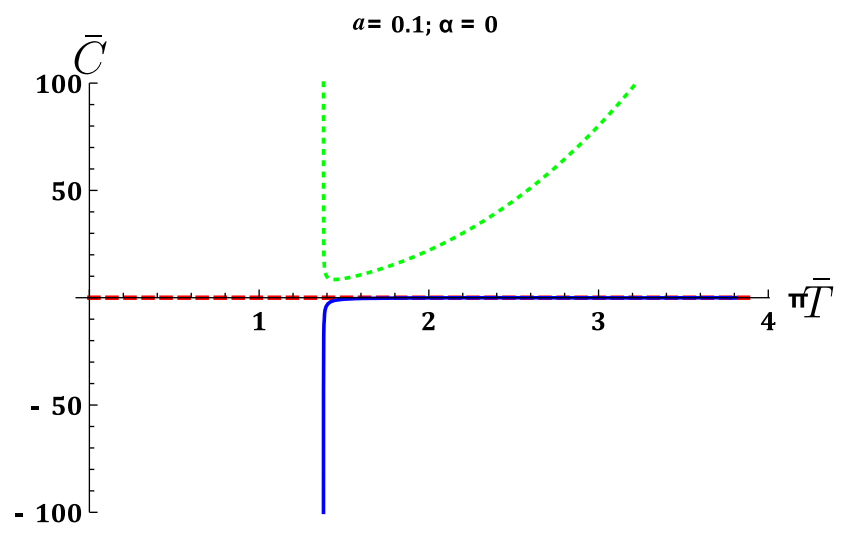

(b)

Fig. 4 a $\bar{r}$ vs $\pi \bar{T}$ plot $\mathbf{b} \bar{C}$ vs. $\pi \bar{T}$ with $\bar{\alpha}=0, \bar{a}=0.1$ for black holes with horizon radii small (red), medium (blue) and large(green)

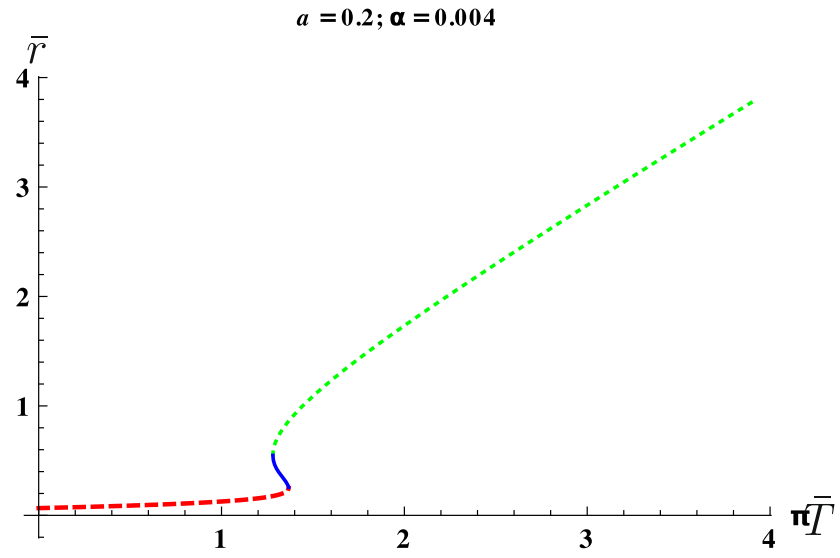

(a)

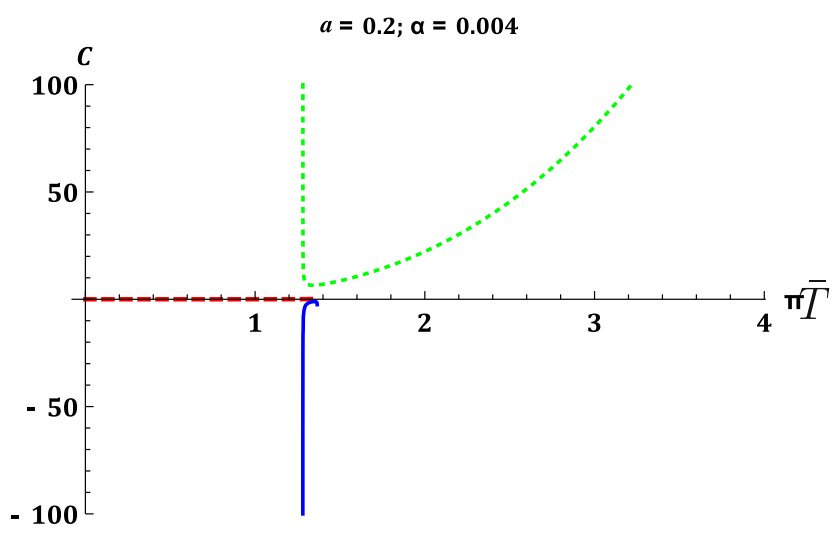

(b)

Fig. 5 a $\bar{r}$ vs $\pi \bar{T}$ plot $\mathbf{b} \bar{C}$ vs. $\pi \bar{T}$ with $\bar{\alpha}=0.004, \bar{a}=0.2$ for black holes with horizon radii small (red), medium (blue) and large(green)

the small black hole remains thermodynamically stable till $\bar{T}<\bar{T}_{1}$, which is consistent with the analysis of the specific heat. As $\bar{T}$ reaches $\bar{T}_{1}$, the other two black holes appear. At any temperature, a comparison of free energies shows the black hole with intermediate horizon radius is not thermodynamically favoured. For $\bar{T}>\bar{T}_{c}$, a critical temperature which is less than $\bar{T}_{2}$, the small black hole has a free energy greater than that of the large black hole and therefore, the large black hole continues to be the thermodynamically favoured configuration for the range of temperature $\bar{T}>\bar{T}_{c}$.

In the Fig. $6 \mathrm{~b}$, we have plotted the free energy vs. the temperature for $\bar{\alpha}=.001, \bar{a}=0.2$. One noticeable difference that happens here is that the free energy for the small black hole is positive up to a certain temperature and then it becomes negative. On the other hand the free energy for the medium and large black holes is negative for any temperature. Except these differences other behaviour are same as previous case.
In the Fig. 7, we have plotted the free energy vs. the scaled horizon radius for two different sets: (a) $\bar{a}=0.1, \bar{\alpha}=0.001$ and (b) $\bar{a}=0.1, \bar{\alpha}=0.01$. As one can observe from these plots at $\bar{r}=0$ the free energy is positive. As the radius of the black hole increases, the free energy decreases and becomes minimum. As we increase $\bar{r}$ further, the free energy starts increasing, becomes positive and reaches a maximum value at a certain radius. For further increase of the horizon radius, the free energy becomes negative and keeps on decreasing. We can associate again free energy of $\bar{r}=0$ as free energy of AdS configuration, the local minima of the free energy as the small black hole free energy and the negative free energy for the large $\bar{r}$ is related to the large black hole. The maxima in the free energy corresponds to the unstable black hole. From the consideration of free energy, AdS configuration is not a stable configuration and small and large black hole can be stable configuration only. 


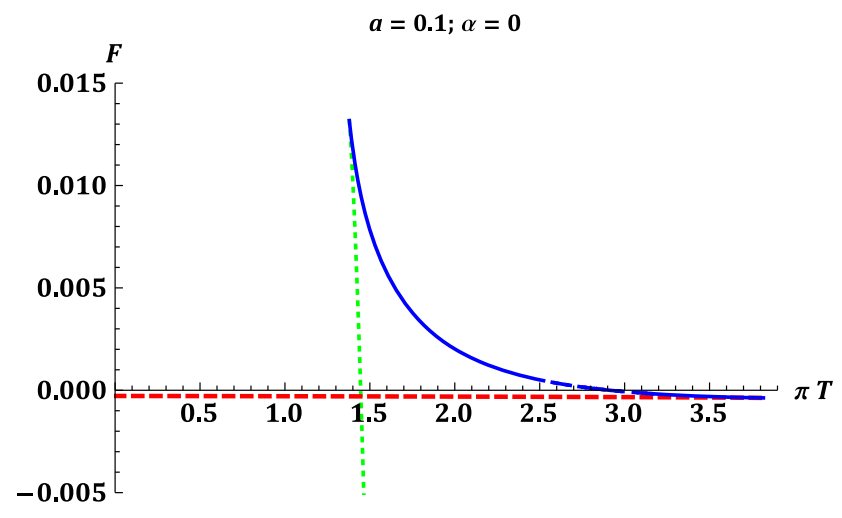

(a)

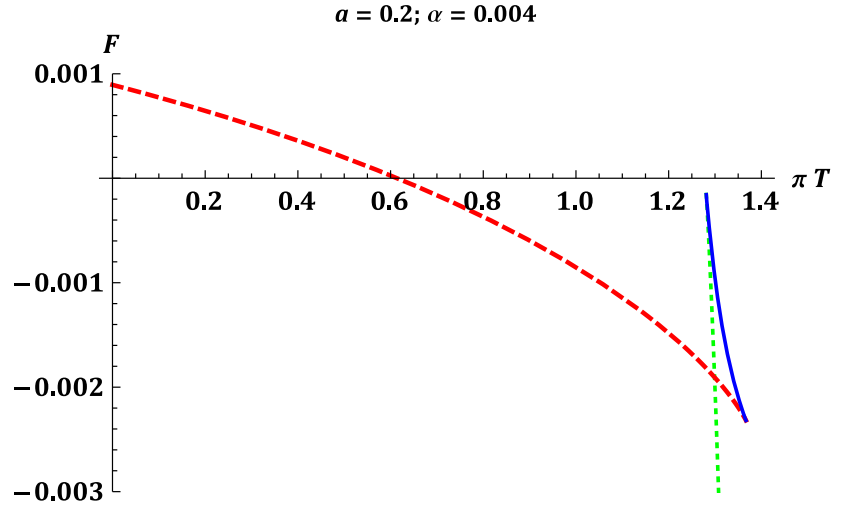

(b)

Fig. 6 Free energy is plotted against temperature: black holes with horizon radii small, medium and large are plotted in red, blue and green. a $\bar{\alpha}=0, \bar{a}=0.1, \mathbf{b} \bar{\alpha}=0.004, \bar{a}=0.2$

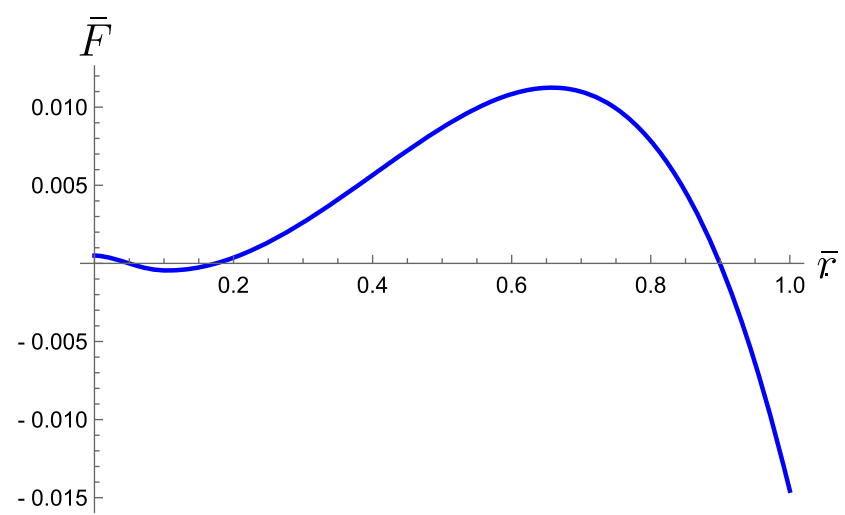

(a)

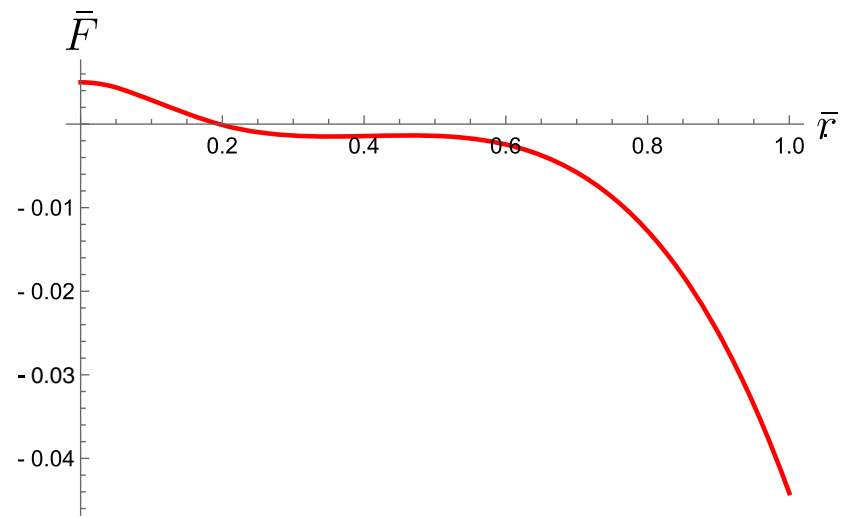

(b)

Fig. 7 Free energy is plotted against horizon radius: $\mathbf{a} \bar{a}=0.1$ and $\bar{\alpha}=0.001$ and plot $\mathbf{b}$ is for $\bar{a}=0.1, \bar{\alpha}=0.01$ and $l=1$

We consider the Landau function which is constructed around the critical point to investigate about stability of different solutions and is given by:

$\bar{G}=\frac{3 l^{2} \bar{r}^{4}-4 \pi l^{3} \bar{r}^{3} T+3 l^{2} \bar{r}^{2}-2 l^{2} \bar{a} \bar{r}-48 \pi l^{3} \bar{\alpha} \bar{r} T+6 \bar{\alpha} l^{2}}{12}$.

We consider four different temperatures and have plotted Landau function with respect to scaled horizon in Fig. 8a with $\bar{a}=0.1$ and $\bar{\alpha}=0.001$. The temperatures we consider are $\bar{T}_{0}=0.42$ (red curve), $\bar{T}_{1}=0.44$ (green curve), $\bar{T}_{c}=0.4545$ (blue curve) and $\bar{T}_{c 1}=0.4568$ (pink curve) such that $\bar{T}_{0}<\bar{T}_{1}<\bar{T}_{c}<\bar{T}_{c 1}$. For temperature $\bar{T}_{0}$, only one minimum of Landau function at finite $\bar{r}$ exists, that corresponds to the black hole solution with small horizon radius. With rise of temperature two more extrema nucleates at temperature $T_{1}$. The maxima of the Landau function corresponds to unstable black hole and minima corresponds to the large black hole. As we increase the temperature further at temperature $T_{c}$ the Landau function associated with the black holes having smallest and largest radii become equal. Above $T_{c}$, the Landau function for large black hole becomes lower than that of the small black hole indicating stability of the small black hole at low temperature and of the large black hole at high temperature. It also indicates that there will be a transition between the small and large black hole at $T=T_{c}$ similar to Hawking-Page phase transition. In Fig. 8b, we plot the Landau function against scaled horizon radius for $\bar{a}=0.1$ and $\bar{\alpha}=0.01$. We observed that there is only one minima at finite $\bar{r}$ corresponds to existence of one stable black hole solution from which one may conclude that pure AdS is not a stable configuration at any temperature.

\section{Quark-antiquark distance}

As pointed out in the introduction, it is interesting to examine the phase of the dual model with reference to the confinement. For that purpose, we consider a string configuration having its endpoints on the boundary and elongated in the 


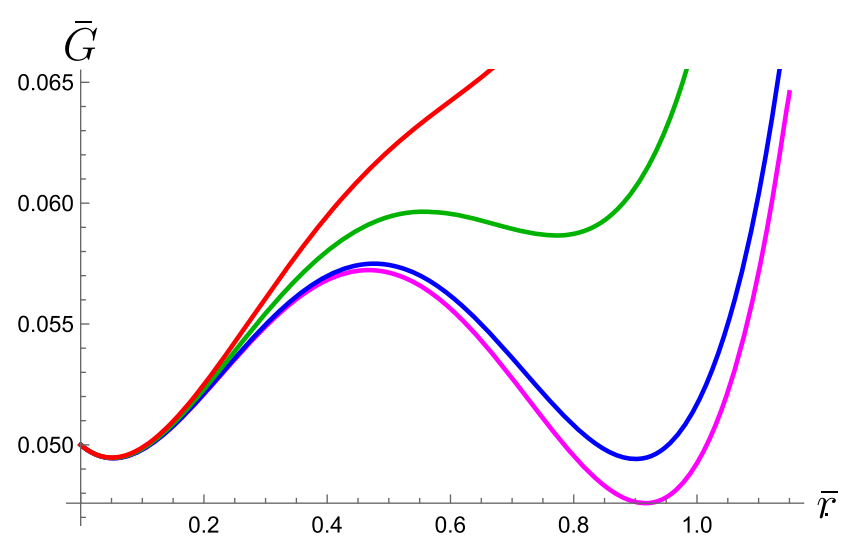

(a)

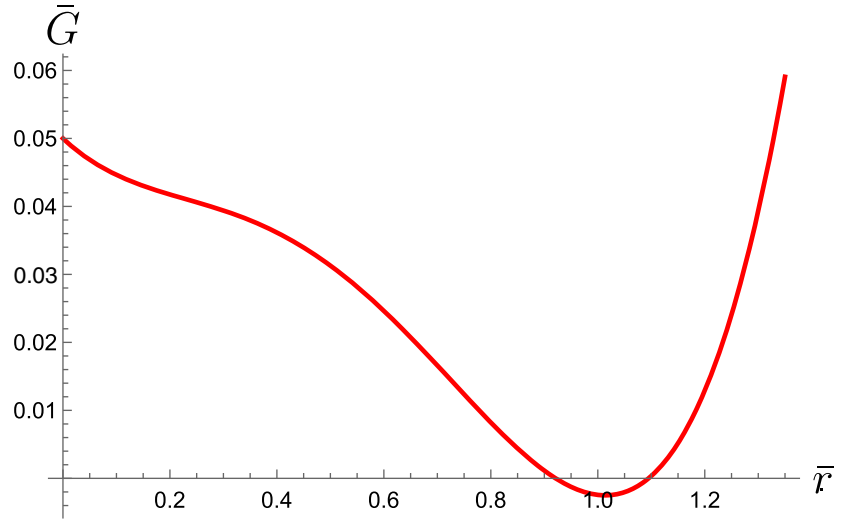

(b)

Fig. 8 Landau function vs. $\bar{r}$ for four different temperatures: $\bar{T}_{0}=0.42$ (red), $\bar{T}_{1}=0.44$ (green), $\bar{T}_{c}=0.4545$ (blue) $\bar{T}_{c 1}=0.4568$ (pink) ; $\bar{T}_{0}<\bar{T}_{1}<\bar{T}_{c}<\bar{T}_{c 1} \mathbf{a} \bar{a}=0.1$ and $\bar{\alpha}=0.001, \mathbf{b}$ is for $\bar{a}=0.1, \bar{\alpha}=0.01$ and $l=1$

bulk. There may be two such configurations: a straight string from boundary to horizon or a U-shaped string where the string is hanging from the boundary, with its tip located at $u_{0}$, but not touching the horizon.

We will denote the distance between the two endpoints by $L$, which is the quark-antiquark distance associated with the bound state in the boundary theory. There is an upper bound $L_{S}$ of the quark-antiquark separation $L$. Every $L, 0<$ $L<L_{s}$ corresponds to two U-shaped strings with tip $u_{0}$ being nearer to and farther from the horizon. As $L$ increases, these two configurations approach each other and they merge at $L=L_{S}$, when the quark-antiquark distance reaches the screening length.

Quark-antiquark distance for a bound state can be obtained by considering a probe string in the black hole spacetime. The Nambu-Goto world sheet action for such an open string is,

$S_{N G}=-\frac{1}{2 \pi \alpha^{\prime}} \int d^{2} \sigma \sqrt{-\operatorname{det}\left(h_{\beta \gamma}\right)}$,

where $\frac{1}{2 \pi \alpha^{\prime}}$ is the string tension. The string tension is related to $\lambda$, the "t Hooft coupling in the dual SYM gauge theory [22],

$\sqrt{\lambda}=\frac{l^{2}}{\alpha^{\prime}}$.

The present case being a bottom-up approach, we do not have a precise description of the dual boundary theory. Nevertheless we can expect that the $\lambda$ will play an analogous role. We will assume that the above Nambu-Goto action is a valid action for our problem and corrections ensuing from coupling to the further bulk fields will not qualitatively modify the essential result.

For convenience, we will use $u$ rather than $r$, where $r=\frac{l^{2}}{u}$ so that $\mathrm{u}=0$ is the boundary and the horizon occurs at $u_{h}$. The metric tensor of the black hole solution of the equation (4) reduces to the form as,

$$
\begin{gathered}
d s^{2}=f(u)\left[-h(u) d t^{2}+d x^{2}+d y^{2}+d z^{2}+\frac{d u^{2}}{h(u)}\right], \\
\text { where, } f(u)=\frac{l^{2}}{u^{2}} \text { and } h(u)=\frac{u^{2}}{l^{2}}+\frac{1}{4 \bar{\alpha}} \\
\left(1-\sqrt{1-8 \bar{\alpha}+\frac{32 \bar{\alpha} M u^{4}}{l^{6}}+\frac{16 \bar{a} \bar{\alpha} u^{3}}{3 l^{3}}}\right)
\end{gathered}
$$

and the radius of the horizon, $u_{h}$, can be obtained by solving the equation,

$$
\begin{aligned}
& h\left(u_{h}\right)=\frac{u_{h}^{2}}{l^{2}} \\
& +\frac{1}{4 \bar{\alpha}}\left(1-\sqrt{1-8 \bar{\alpha}+\frac{32 \bar{\alpha} M u_{h}^{4}}{l^{6}}+\frac{16 \bar{a} \bar{\alpha} u_{h}^{3}}{3 l^{3}}}\right)=0 .
\end{aligned}
$$

We will consider, the end points of the open string corresponding to $Q \bar{Q}$ pair are located at $x= \pm \frac{L}{2}$ respectively and is elongated in the bulk space time with the symmetry around $x=0$.

With this background, we evaluate the Nambu-Goto action, (23). We choose: $\sigma^{0}=t, \sigma^{1}=x$ (static gauge) and the metric induced on the worldsheet, given by $h_{\beta \gamma}=$ $\partial_{\beta} X^{\mu} \partial_{\gamma} X^{\nu} g_{\mu \nu}$ becomes,

$d s^{2}=f(u)\left[-h(u) d t^{2}+\left\{1+\frac{u^{\prime 2}}{h(u)}\right\} d x^{2}\right]$.

After $t$ integration is carried out, the Nambu-Goto action becomes

$S_{N G}=-\frac{\mathcal{T}}{2 \pi \alpha^{\prime}} \int_{-L / 2}^{L / 2} d x f(u) \sqrt{h(u)\left(1+\frac{u^{\prime 2}}{h(u)}\right)}$. 


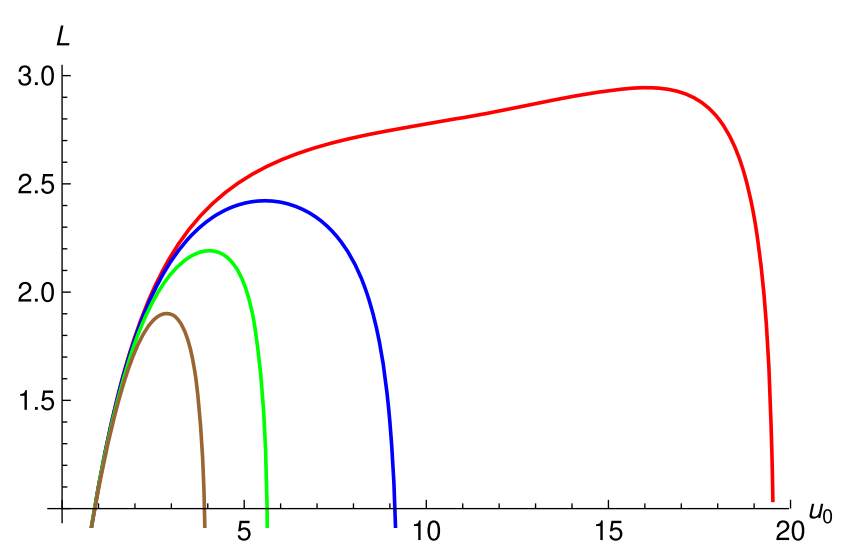

(a)

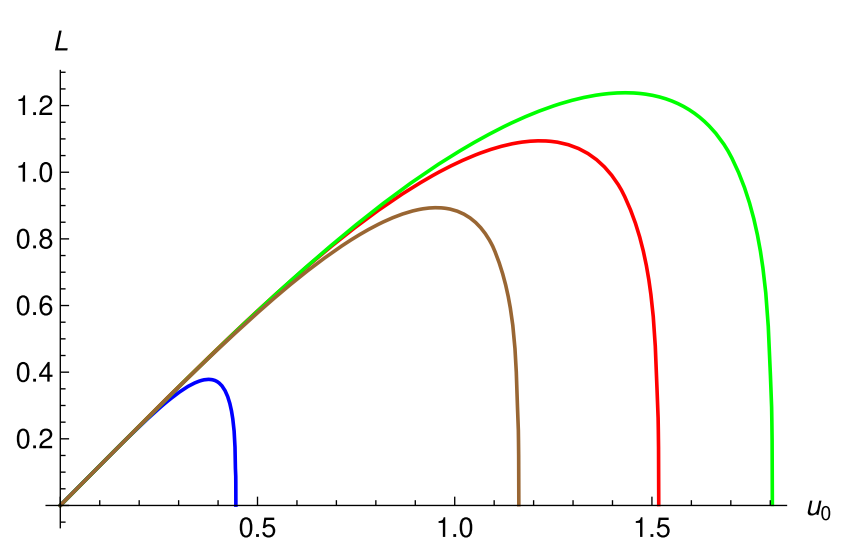

(b)

Fig. $9 \mathrm{Q} \bar{Q}$-distance vs. $u_{0}: \bar{a}=.1, \bar{\alpha}=.001, T=T_{1}$ (red), $T=T_{2}$ (blue); $\bar{a}=.2, \alpha=.004, T=T_{1}$ (green), $T=T_{2}$ (brown); black holes with radius a small, b large

The equations of motion for the embedding coordinate $u(x)$, ensuing from the above action is given by

$u^{\prime}(x)=-\sqrt{h(u)\left(\frac{f(u)^{2} h(u)}{f\left(u_{0}\right)^{2} h\left(u_{0}\right)}-1\right)}$,

implying the string is extended towards the horizon up to a turning point $u=u_{0}$ and goes back to the boundary in a symmetric manner with $u(0)=u_{0}$. The distance between the two end points of the string can be obtained as

$$
\begin{aligned}
L & =\int_{-L / 2}^{L / 2} d x=2 \int_{0}^{u_{0}} \frac{d u}{u^{\prime}} \\
& =2 \int_{0}^{u_{0}} d u\left[h(u)\left(\frac{f(u)^{2} h(u)}{f\left(u_{0}\right)^{2} h\left(u_{0}\right)}-1\right)\right]^{-\frac{1}{2}},
\end{aligned}
$$

where $u_{0}$ is the upper bound on the location of the tip of the string that is extended towards horizon.

We have plotted the quark-antiquark separation $L$ with respect to $u_{0}$, the upper bound on the location of the tip of the string extended towards the horizon in Fig. 9. One can observe from the figure, as the tip located at $u_{0}$ gets closer to horizon, quark-antiquak separation increases. It becomes maximum when $u_{0}$ gets extremely near to the horizon and once it touches the horizon, $L$ becomes zero. This fact can be interpreted as a breakdown of the U-shaped string configuration to two parallel straight string configuration which corresponds to unbound state of $Q \bar{Q}$ pair. This happens in case of the small as well as the large black hole. It suggests that confined state might not be present in the dual theory living on the boundary, as expected in gravity analysis.

\section{Quark-antiquark potential}

In the present section we are interested to study free energy associated with the heavy quark-antiquark pair of the dual theory living on the boundary. The Wegner-Wilson loop encodes the free energy associated with a quark-antiquark pair [22],

$\left\langle W\left(\mathcal{C}_{L, \mathcal{T}}\right)\right\rangle \sim \exp \left[-i F_{Q \bar{Q}}(L) \mathcal{T}\right], \quad \mathcal{T} \rightarrow \infty$,

where $F_{Q \bar{Q}}(L)$ is the $Q \bar{Q}$ free energy. It depends on the temperature as the expectation value is for a thermal state. Since in the present discussion we will consider the range of $L$ only up to a screening distance $L_{S}$, the free energy $F_{Q \bar{Q}}$ will agree with the quark-antiquark potential $V_{Q \bar{Q}}$ in our case.

According to holographic principle, Nambu-Goto action of an open string given in equation (23) is related to the Wegner-Wilson loop where $\mathcal{C}$, the contour of integration is identified with open string worldsheet boundary. The on-shell string action then is related to expectation value,

$\langle W(\mathcal{C})\rangle \sim \exp \left[i S_{N G}(\mathcal{C})\right]$

where $S_{N G}(\mathcal{C})$ is the on-shell Nambu-Goto action of the string whose expression is given in (28). Comparing (31) and (32), we obtain

$F_{Q \bar{Q}}(L) \sim-\frac{S_{N G}(\mathcal{C})}{\mathcal{T}}$.

$F_{Q \bar{Q}}(L)$, free energy for quark-antiquark can be obtained by substituting (29) in (28). As has been discussed elaborately in [22], computation of $S_{N G}$ in general gives rise to divergences and this expression needs to be renormalised. We follow the same renormalisation prescription as given in [22] and obtain a renormalised expression for the quark-antiquark free energy, given by 


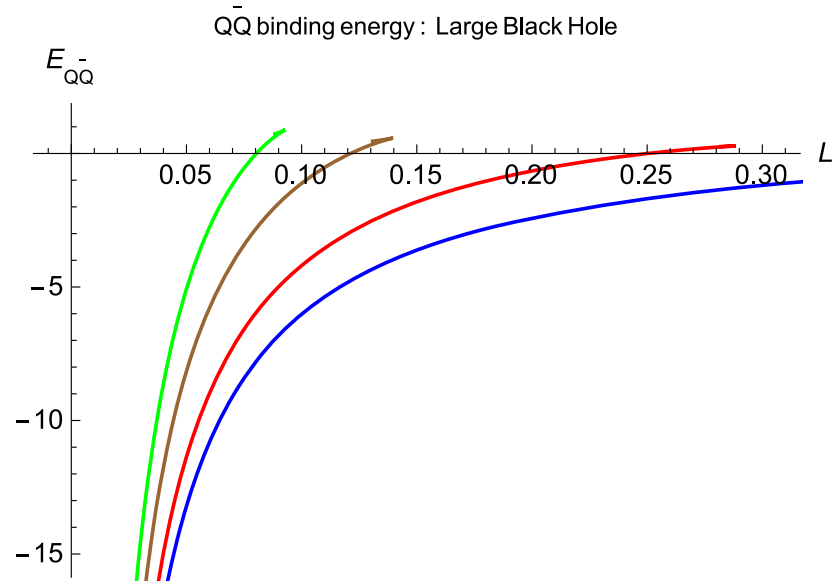

(a)

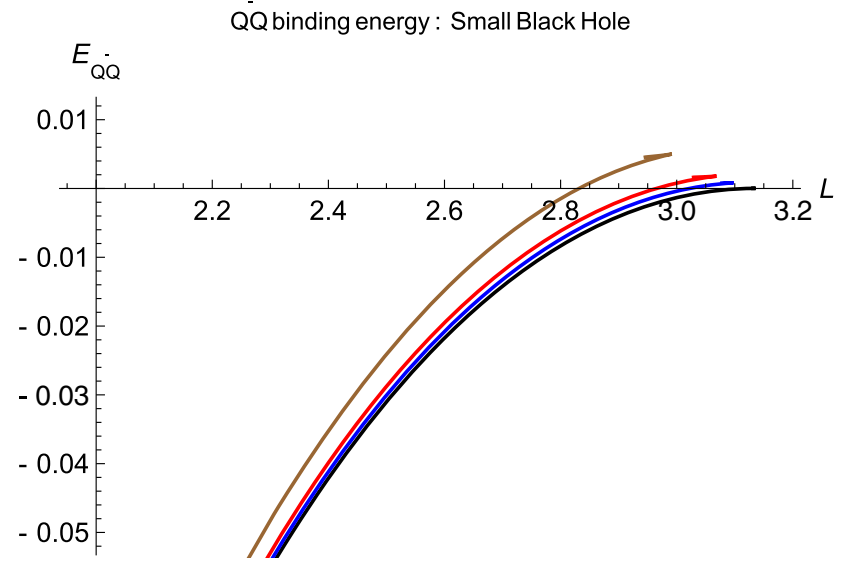

(b)

Fig. $10 \bar{a}=.05$; a $Q \bar{Q}$ binding energy for the large black hole for temperatures, $\mathrm{T}=3$ (green), 2 (brown), 1 (red), 0.5 (blue), b $Q \bar{Q}$ binding energy for the small black hole for temperatures, $\mathrm{T}=2$ (brown), 1 (red), 0.5 (blue), .01 (black)

$$
\begin{aligned}
& \frac{\pi F_{Q \bar{Q}}\left(u_{0}\right)}{\sqrt{\lambda}}=\int_{0}^{u_{0}} d u \\
& {\left[f(u) \sqrt{\frac{f(u)^{2} h(u)}{f(u)^{2} h(u)-f\left(u_{0}\right)^{2} h\left(u_{0}\right)}}-\frac{1}{u^{2}}\right]-\frac{1}{u_{0}} .}
\end{aligned}
$$

Usually quark-antiquark binding energy can be obtained from U-shaped string action with action of two straight strings from boundary to horizon subtracted from it, which is given by [22]

$$
\begin{aligned}
& \frac{\pi E_{Q \bar{Q}}\left(u_{0}\right)}{\sqrt{\lambda}}=\int_{0}^{u_{0}} d u \\
& {\left[f(u) \sqrt{\frac{f(u)^{2} h(u)}{f(u)^{2} h(u)-f\left(u_{0}\right)^{2} h\left(u_{0}\right)}}-1\right]-\int_{u_{0}}^{u_{h}} f(u) d u .}
\end{aligned}
$$

In what follows, in both the cases of small and large black hole we can obtain free energy and binding energy for the quark-antiquark potential. We begin with black holes that has been obtained without the higher derivative coupling. Some result has already appeared in [12] and here we have a more elaborate analysis. If we drop the higher derivative terms, metric functions are [12]

$$
\begin{aligned}
& f(u)=\frac{1}{u^{2}}, \quad h(u)=1-\left(\frac{u}{u_{h}}\right)^{4}+u^{2}\left[1-\left(\frac{u}{u_{h}}\right)^{2}\right] \\
& -\frac{2 \bar{a}}{3} u^{3}\left(1-\frac{u}{u_{h}}\right),
\end{aligned}
$$

where we have traded $M$ for horizon radius $u_{h}$. Here and in the rest of the calculation, we set $l=1$
We have substituted these expressions in the general formula for the free energy and the binding energy for the quarkantiquark pair derived in (34) and (35) respectively. Since the integration cannot be carried out to obtain expressions in terms of known analytic functions, we have evaluated them numerically. We obtain the quark-antiquark separation from the expression (30) as a function of $u_{0}$, the closest approach of the string worldsheet towards the horizon. It turns out that as $u_{0}$ varies there is a maximum value of the quark-antiquark separation, which depends on the temperature as well.

In order to find out the thermodynamically stable quarkantiquark bound states we have plotted the binding energy versus the quark-antiquark separation for both the black holes in the Fig. 10. One may observe that the binding energy of the bound state for large black hole is smaller than the small black hole background indicating that the bound states are more stable in the background of large black hole. One can also notice that for both the cases, after a certain value of separation distance the binding energy of the pair is getting positive value. Therefore the bound state exist up to an upper value of separation distance which is called screening length $L_{s}$ and that is finite. The screening length decreases with the increase of temperature and bound state becomes more unstable at higher temperature. We compare the screening length for small and large black hole background and find screening length is larger in small black hole configuration showing it admits larger separation, but in none of the cases, it can go to infinity or can be interpreted as deconfined phase. In Fig. 11 we have analysed the same features for different values of string cloud density by keeping temperature fixed. The qualitative behaviour remains unchanged and here string cloud density plays a role analogous to the temperature. Finally we can conclude that deconfined state of quark and antiquark is the stable configuration. 


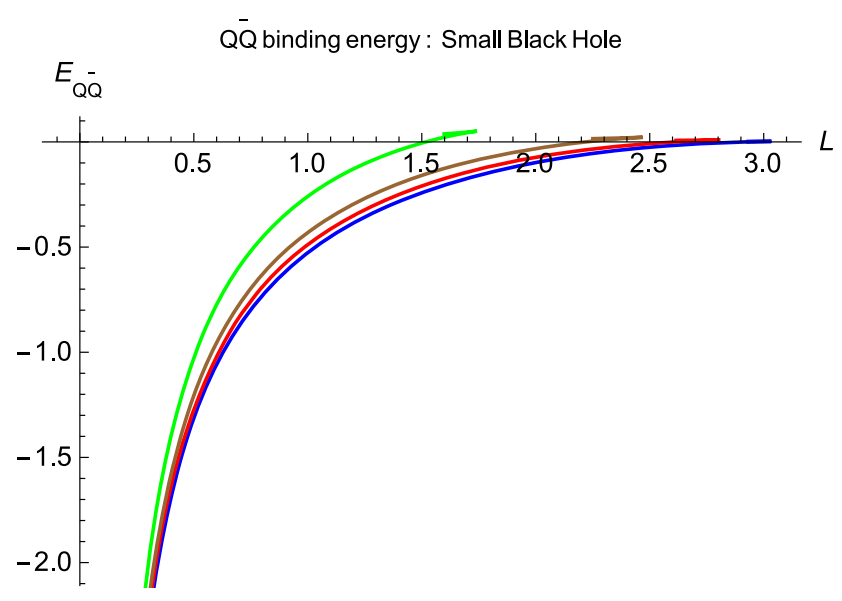

(a)

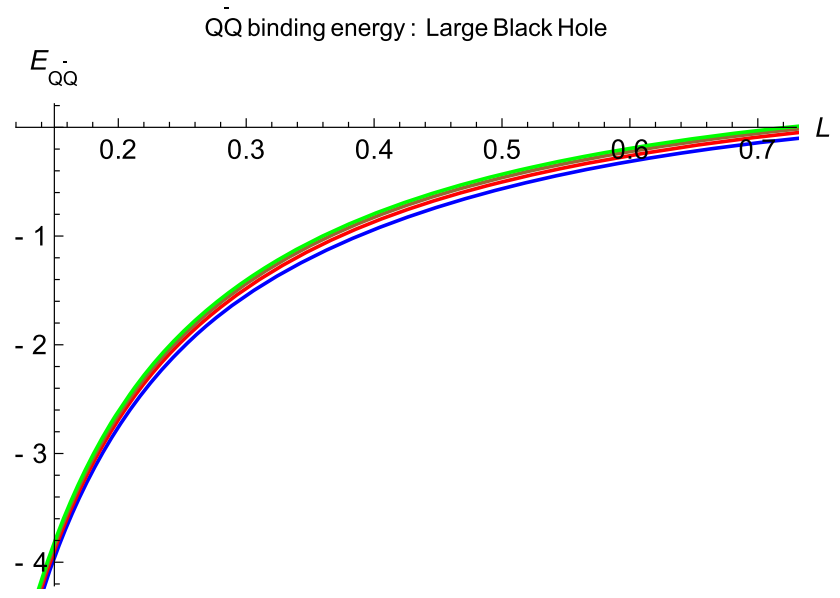

(b)

Fig. $11 Q \bar{Q}$ binding energy for different $\bar{a}$ values, $\bar{a}=.4$ (green), .3 (brown), .2 (red), .1 (blue) a small black hole, $T=1, \mathbf{b}$ large black hole, $T=0.4$;

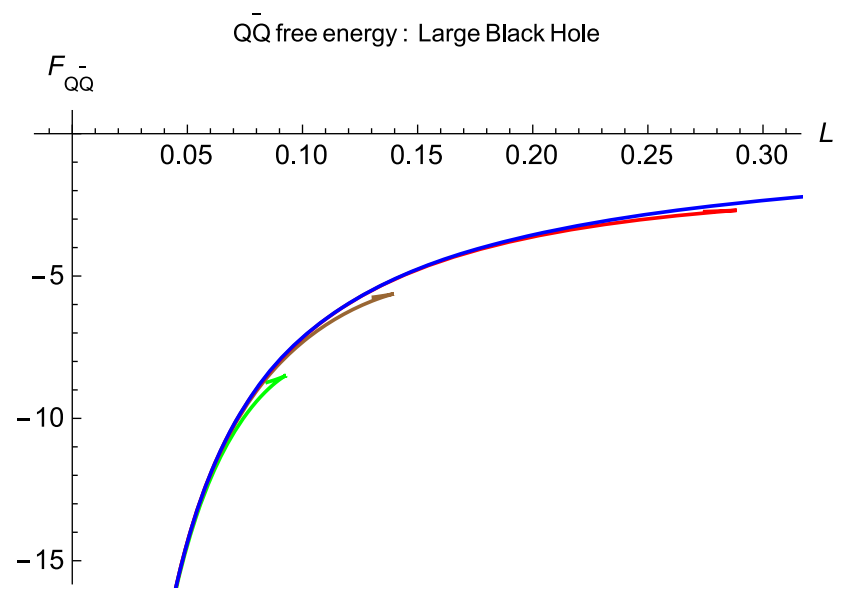

(a)

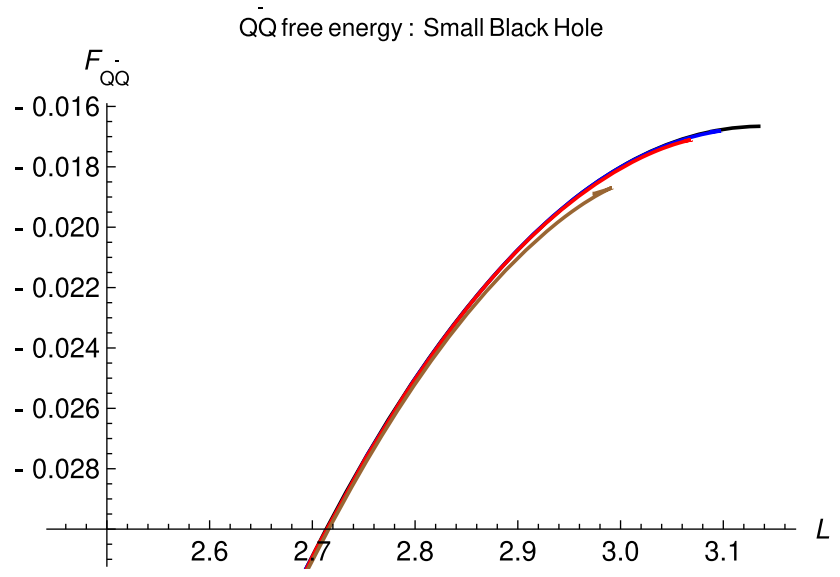

(b)

Fig. $12 \bar{a}=.05 ; Q \bar{Q}$ free energy vs. L: a large black hole with T = 3 (green), 2 (brown), 1 (red), 0.5 (blue), b small black hole with T = 2 (brown), 1 (red), 0.5 (blue), .01 (black)

The quark-antiquark free energy obtained from (34) are numerically evaluated for both the black holes in the same range of temperatures and are plotted with respect to the quark-antiquark separation in Fig. 12. Comparing with the binding energy plotted in Fig. 10, one can observe that with variation of the temperature the free energy associated with quark-antiquark pair does not vary much, though there is a reasonable difference in the binding energy, mostly arising from the subtraction of the energy due to the straight strings.

Modification due to higher derivative terms can be studied by considering black hole solution obtained from EinsteinGauss-Bonnet action as discussed in the previous sections. Once higher derivative terms are taken into account, the metric is given in (4) and (5). In terms of the general form of metric given in (25) the metric functions become

$$
\begin{aligned}
& f(u)=\frac{1}{u^{2}} \\
& h(u)=u^{2}+\frac{1}{4 \bar{\alpha}} \\
& {\left[1-\sqrt{\left.1-8 \bar{\alpha}\left(1-\frac{u^{4}}{u_{h}^{4}}\right)+\frac{16 \overline{\bar{\alpha}} \bar{\alpha}}{3} u^{3}\left(1-\frac{u}{u_{h}}\right)+32 \bar{\alpha} u^{4}\left(\frac{1}{4 u_{h}^{2}}+\frac{\bar{\alpha}}{2}\right)\right]},\right.}
\end{aligned}
$$

where, as usual, we have traded mass parameter $M$ for the horizon radius $u_{h}$.

Substituting the above expressions in the general formula for the binding energy and the separation for the quarkantiquark pair given in (34) and (30) respectively, we have plotted the binding energy against $L$. We consider four different values of $\bar{\alpha}$, the coefficient of higher derivative coupling in the plots for both the black holes as given in Fig. 13. 


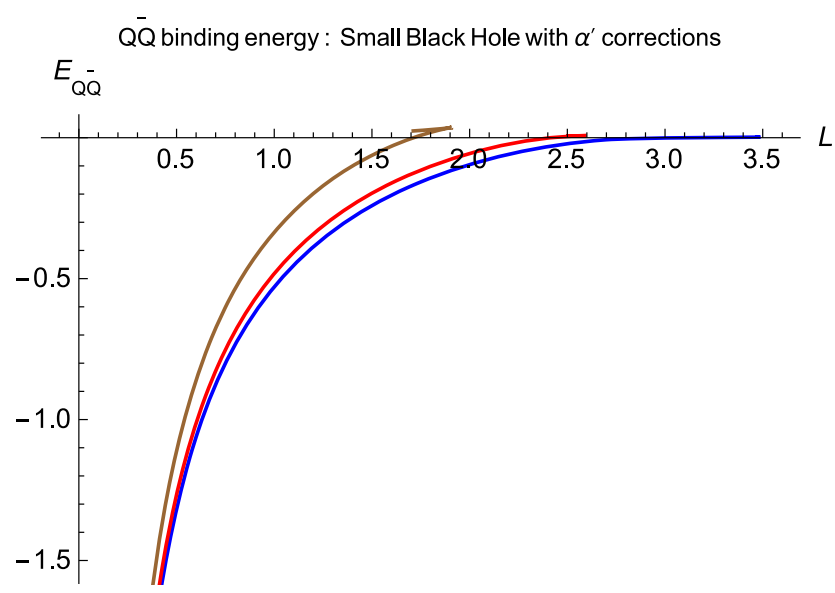

(a)

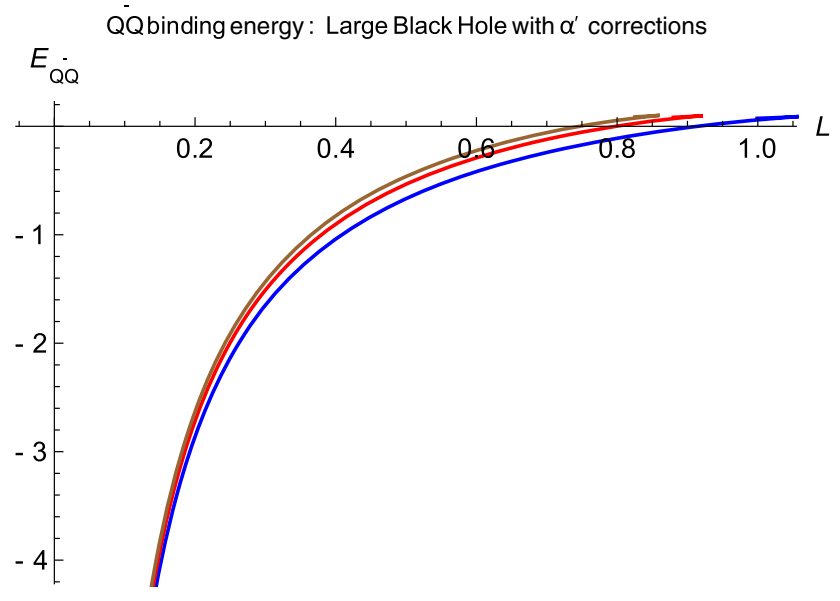

(b)

Fig. $13 Q \bar{Q}$ binding energy vs. L for $\bar{a}=.05 ; T=.441 ; \bar{\alpha}=.001$ (blue), .004 (red), .0082 (brown) a small black hole, b large black hole

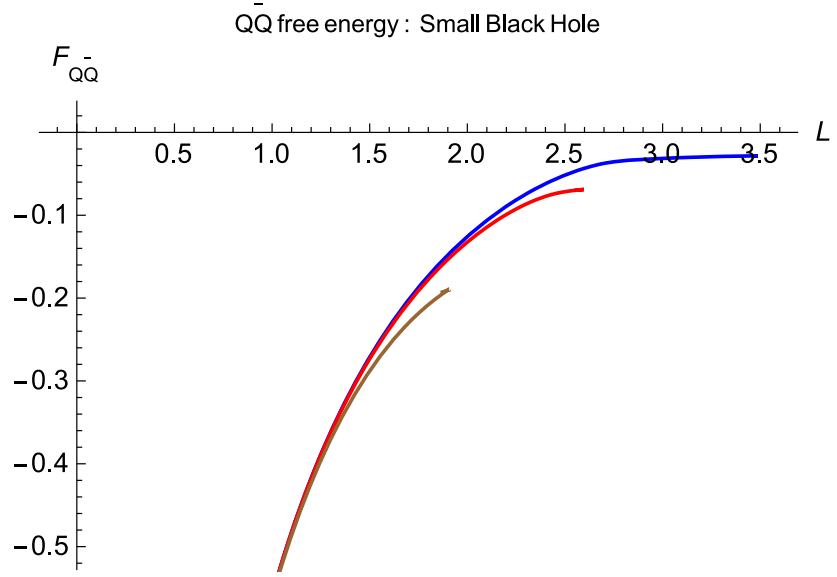

(a)

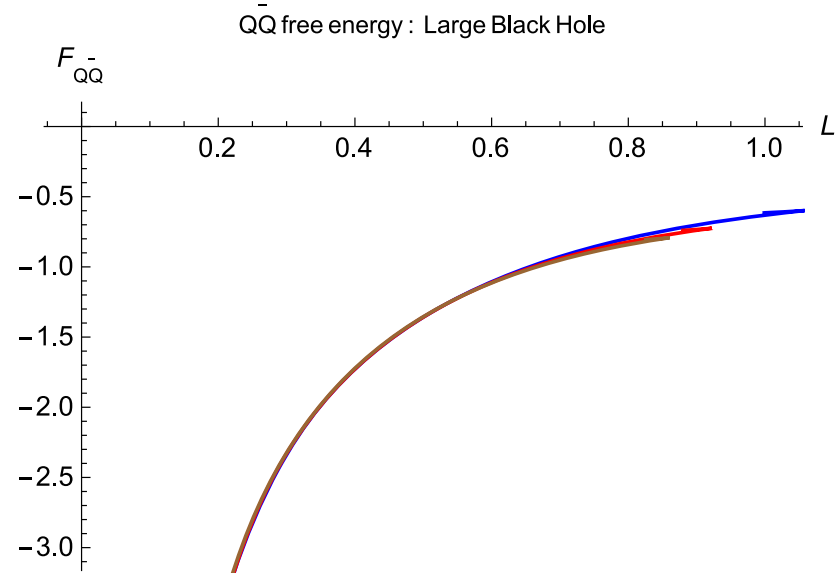

(b)

Fig. $14 Q \bar{Q}$ free energy vs. L for $\bar{a}=.05 ; T=.441 ; \bar{\alpha}=.001$ (blue), .004 (red), .0082 (brown) a small black hole, b large black hole

Since, as the $\bar{\alpha}$ varies, the critical temperature keeps on changing, we have chosen suitable values of the temperatures for the two different black holes. As the plots show, with the increase of the coefficient of the higher derivative terms the binding energy increases making the quark-antiquark bound state less stable. Furthermore, for a fixed amount of binding energy the length of the maximum separation between the quark and the antiquark decreases. This indicates, with addition of subleading corrections in 't Hooft coupling, the $Q \bar{Q}$ bound states will become loosely bound favouring deconfinement. Both the small and the large black holes share this feature. Though we have given the plot only for a single temperature in each case, this feature qualitatively persists with variation of the temperature.

At last we have studied the quark-antiquark free energy for both the black holes. We plot the analysis in Fig. 14 and observe that qualitative features are same as without higher derivative term.

\section{Summary}

We have considered solutions in the Einstein Gauss-Bonnet gravity along with an external string clouds. As happened in the Einstein Gauss Bonnet gravity [18], the theory admits three different black holes in presence of string clouds. As the density of string cloud increases and/or the higher derivative correction dominates, the parameter region for which one gets the three solutions shrinks. Beyond the critical values of string cloud density or higher derivative correction, the theory admits only a single solution. 
The analysis of the specific heat associated with the solutions implies that the medium size black hole is unstable, while the other two solutions are thermodynamically stable. From the free energy consideration we find at a higher temperature the thermodynamically faouvored configuration is the black hole with largest horizon radius. We have analysed Landau function to examine possible transitions among these solutions.

Using holographic duality, we examine the quarkantiquark bound states. We find such bound states exist up to a distance of screening length, beyond which they get separated, indicating the dual theory is in a deconfined phase. The screening length turns out to be larger for small black hole than that in large black hole, though it is finite and cannot be extended indefinitely as happened in a confined phase. From the study of quark antiquark binding energy, we find it decreases as the string cloud density and the higher derivative correction becomes larger. This may be interpreted as, in presence of large number of quarks in the background, the quark-antiquark bound states will be loosely bound. A similar effect appears as the subleading corrections in ' $\mathrm{t}$ Hooft coupling becomes increasingly dominant.

It may be interesting to consider further extension of this model. In particular one can consider the charged black hole solution in presence of string clouds and examine the different phases admitted by such theory.

Data Availability Statement This manuscript has no associated data or the data will not be deposited. [Authors' comment: The numerical computations are done using "Mathematica". The output data are plotted in the appropriate figures. The programs, if required, can be obtained by contacting any of the authors.]

Open Access This article is licensed under a Creative Commons Attribution 4.0 International License, which permits use, sharing, adaptation, distribution and reproduction in any medium or format, as long as you give appropriate credit to the original author(s) and the source, provide a link to the Creative Commons licence, and indicate if changes were made. The images or other third party material in this article are included in the article's Creative Commons licence, unless indicated otherwise in a credit line to the material. If material is not included in the article's Creative Commons licence and your intended use is not permitted by statutory regulation or exceeds the permitted use, you will need to obtain permission directly from the copyright holder. To view a copy of this licence, visit http://creativecomm ons.org/licenses/by/4.0/.

Funded by SCOAP ${ }^{3}$.

\section{References}

1. J.M. Maldacena, The large $\mathrm{N}$ limit of superconformal field theories and supergravity. Adv. Theor. Math. Phys. 2, 231 (1998). arXiv:hep-th/9711200

2. J.M. Maldacena, The large $\mathrm{N}$ limit of superconformal field theories and supergravity. Int. J. Theor. Phys. 38, 1113 (1999)

3. E. Witten, Anti-de Sitter space and holography. Adv. Theor. Math. Phys. 2, 253 (1998). arXiv:hep-th/9802150
4. E. Witten, Anti-de Sitter space, thermal phase transition, and confinement in gauge theories. Adv. Theor. Math. Phys. 2, 505 (1998). arXiv:hep-th/9803131

5. S. Hawking, D. Page, Commun. Math. Phys. 87, 577 (1983)

6. E.I. Guendelman, A. Rabinowitz, The Gravitational field of a hedgehog and the evolution of vacuum bubbles. Phys. Rev. D 44, 3152 (1991). https://doi.org/10.1103/PhysRevD.44.3152

7. A. Karch, E. Katz, Adding flavor to AdS / CFT. JHEP 0206, 043 (2002). https://doi.org/10.1088/1126-6708/2002/06/ 043. arXiv:hep-th/0205236

8. M. Headrick, Hedgehog black holes and the Polyakov loop at strong coupling. Phys. Rev. D 77, 105017 (2008). https://doi.org/10.1103/ PhysRevD.77.105017. arXiv:0712.4155 [hep-th]

9. F. Bigazzi, A.L. Cotrone, J. Mas, D. Mayerson, J. Tarrio, D3-D7 quark-gluon plasmas at finite baryon density. JHEP 1104, 060 (2011). https://doi.org/10.1007/JHEP04(2011)060. arXiv: 1101.3560 [hep-th]

10. S.P. Kumar, Heavy quark density in $\mathrm{N}=4 \mathrm{SYM}$ : from hedgehog to Lifshitz spacetimes. JHEP 1208, 155 (2012). https://doi.org/10. 1007/JHEP08(2012)155. arXiv:1206.5140 [hep-th]

11. S. Chakrabortty, Dissipative force on an external quark in heavy quark cloud. Phys. Lett. B 705, 244 (2011). https://doi.org/10. 1016/j.physletb.2011.09.112. arXiv:1108.0165 [hep-th]

12. T.K. Dey, Phase transition of AdS-Schwarzschild black hole and gauge theory dual in the presence of external string cloud. Int. J. Mod. Phys. A 33(33), 1850193 (2018). arXiv:1711.07008 [hep-th]

13. E. Herscovich, M.G. Richarte, Black holes in Einstein-GaussBonnet gravity with a string cloud background. Phys. Lett. B 689, 192 (2010). https://doi.org/10.1016/j.physletb.2010.04.065. arXiv: 1004.3754 [hep-th]

14. S.G. Ghosh, S.D. Maharaj, Cloud of strings for radiating black holes in Lovelock gravity. Phys. Rev. D 89(8), 084027 (2014). https://doi.org/10.1103/PhysRevD.89.084027. arXiv: 1409.7874 [gr-qc]

15. H. Ghaffarnejad, E. Yaraie, Effects of a cloud of strings on the extended phase space of Einstein-Gauss-Bonnet AdS black holes. Phys. Lett. B 785, 105 (2018). arXiv:1806.06687 [gr-qc]

16. M. Ghanaatian, M. Sadeghi, H. Ranjbari, G. Forozani, Effects of the external string cloud on the Van der Waals like behavior and efficiency of AdS-Schwarzschild black holes in massive gravity. arXiv:1906.00369 [hep-th]

17. H. Ranjbari, M. Sadeghi, M. Ghanaatian, G. Forozani, Critical behavior of AdS Gauss-Bonnet massive black holes in the presence of external string cloud. Eur. Phys. J. C 80(1), 17 (2020). https://doi. org/10.1140/epjc/s10052-019-7592-8. arXiv:1911.10803 [hep-th]

18. R.G. Cai, Gauss-Bonnet black holes in AdS spaces. Phys. Rev. D 65, 084014 (2002). https://doi.org/10.1103/PhysRevD.65.084014. arXiv:hep-th/0109133

19. T.K. Dey, S. Mukherji, S. Mukhopadhyay, S. Sarkar, Phase transitions in higher derivative gravity. JHEP 0704, 014 (2007). https:// doi.org/10.1088/1126-6708/2007/04/014. arXiv:hep-th/0609038

20. Y. Yang, P.H. Yuan, Confinement-deconfinement phase transition for heavy quarks in a soft wall holographic QCD model. JHEP 1512, 161 (2015). https://doi.org/10.1007/JHEP12(2015)161. arXiv:1506.05930 [hep-th]

21. M.W. Li, Y. Yang, P.H. Yuan, Phys. Rev. D 96(6), 066013 (2017). arXiv:1703.09184 [hep-th]

22. C. Ewerz, O. Kaczmarek, A. Samberg, Free energy of a heavy quark-antiquark pair in a thermal medium from AdS/CFT. JHEP 1803, 088 (2018). arXiv:1605.07181 [hep-th] 